\title{
Acupuncture and dry eye: current perspectives. A double-blinded randomized controlled trial and review of the literature
}

This article was published in the following Dove Press journal:

Clinical Ophthalmology

\author{
Deepinder K Dhaliwal' \\ Siwei Zhou' \\ Sandeep S Samudre ${ }^{2}$ \\ Nathan J Lo ${ }^{3}$ \\ Michelle K Rhee ${ }^{4}$ \\ 'Department of Ophthalmology, \\ University of Pittsburgh Medical Center, \\ Pittsburgh, PA, USA; ${ }^{2}$ Department of \\ Ophthalmology, Eastern Virginia Eye \\ Institute, Norfolk, VA, USA; \\ ${ }^{3}$ Department of Medicine, Duke \\ University Hospital, Durham, NC, USA; \\ ${ }^{4}$ Department of Ophthalmology, Icahn \\ School of Medicine at Mount Sinai, \\ New York, NY, USA
}

Purpose: Dry eye disease (DED) is a common disorder that negatively impacts quality of life and vision. Prior studies have shown some benefit of acupuncture for dry eye, but very few have included control group to mitigate placebo effect. This study was designed with a sham acupuncture control group to evaluate true acupuncture treatment effect.

Methods: This is a prospective, randomized, double-blinded, sham-acupuncture-controlled trial. Acupuncture treatment for dry eye was performed as per the Niemtzow Protocol. Twenty-four patients received true acupuncture and twenty-five received sham acupuncture. Treatment efficacy was assessed by the Ocular Surface Disease Index (OSDI) Questionnaire, ocular surface staining, tear flow, tear film break-up time (TBUT), and a general questionnaire. Atmospheric data were collected to control for the effect of atmospheric conditions on symptoms of dry eye.

Results: OSDI scores in the treatment group improved compared to baseline (1 week, $p<0.01,1$ month $p<0.05,3$ months $p<0.05$, and 6 months $p<0.01)$. OSDI scores in the control group improved, but did not reach significance $(p=0.09)$. Secondary outcome measures showed no significant improvement in TBUT, Schiermer's Test, ocular surface grading, or artificial tear application. However, at 3 months, a significant reduction in the frequency of eye closing was observed among participants receiving true acupuncture treatment when compared to baseline $(p=0.002)$. Furthermore, intragroup analysis showed significant reduction in symptoms of discomfort $(p=0.01)$, dryness $(p=0.001)$, scratchiness $(p=0.001)$, and redness $(p=0.01)$ in the true acupuncture group at 3 months.

Conclusion: Both true and sham acupuncture improved OSDI at 1 week after treatment, however, the improvement in OSDI was significantly greater in the true treatment groups than the sham group at 6 months after acupuncture. True acupuncture treatment improved many subjective assessments of dry eye symptoms, however, other common indicators used to objectively assess dry eye (tear flow, corneal staining, TBUT) remained unchanged. While there were trends towards improvement in the sham acupuncture group, this did not reach statistical significant during the study period. This suggests a true treatment effect of acupuncture rather than a placebo effect. Acupuncture can, therefore, be an effective adjunct to routine clinical treatment of dry eye.

Keywords: acupuncture, dry eye, keratoconjunctivitis sicca, sham acupuncture

\section{Introduction}

Dry eye disease (DED), or keratoconjunctivitis sicca, is one of the most common ocular problems in the United States and one of the most frequent reasons for seeking eye care. ${ }^{1,2}$ The prevalence of dry eye is estimated to be between $5 \%$ to
Correspondence: Michelle K Rhee Department of Ophthalmology, Icahn School of Medicine at Mount Sinai, 305 East 55th Street Suite 105, New York, NY 10022, USA

Tel + I 6465432015

Fax + I 7183345449

Email Michelle.rhee@mssm.edu 
$>30 \%$ at various ages. ${ }^{3}$ DED symptoms can be particularly debilitating and include irritation, sandy or gritty feeling, burning or stinging, tiredness, and redness of the eyes. These can have a major negative effect on quality of vision and quality of life. ${ }^{4-6}$ Current treatment consists mostly of symptomatic relief through lifestyle modifications, frequent use of lubricant solutions and artificial tears, topical cyclosporine, topical steroids, lacrimal punctal occlusion, oral tetracyclines, and oral omega- 3 fatty acids. ${ }^{7-9}$ More recent technological advances include Intense Pulsed Light and LipiFlow. ${ }^{10}$ However, even with such treatment, patients often continue to have debilitating symptoms. The pharmacoeconomic analysis postulates reductions in productivity equivalent to an estimated annual cost of US $\$ 6,466$ per person due to DED. ${ }^{11,12}$

Acupuncture originated more than 3,000 years ago in China and is practiced in most of the world for various disease states. ${ }^{13-17}$ Prior studies and case reports have reported acupuncture as an effective treatment for dry eye. ${ }^{18-28}$ However, other studies have suggested either a lack of effect or placebo effect. $^{29,30}$ Very few prior studies compared true acupuncture with a sham acupuncture control group, ${ }^{31,32}$ and thus a placebo effect could not be ruled out. Many of these studies lacked any control group altogether and had poor methodology, making it difficult to draw any strong conclusions about the efficacy of acupuncture for dry eye.

The objective of this study was to improve the current literature in assessing the efficacy of acupuncture for DED by implementing a methodologically sound, prospective, randomized, double-blinded, sham-acupuncture-controlled study, using some of the most widely used clinical indicators in the literature.

\section{Materials and methods}

This randomized control trial received approval from the Institution Review Board of the University of Pittsburgh and was conducted in accordance with the Declaration of Helsinki.

\section{Participants}

A total of 58 subjects were recruited from flyers, a newsletter insert, a telephone-recorded broadcast ad, and from the investigator's clinic at the University of Pittsburgh Medical Center (UPMC) Eye Center. Inclusion and exclusion criteria are shown in Table 1. Written informed consent was obtained from each patient prior to enrollment in the study. All women of child-bearing age were required to take a urine sample pregnancy test prior
Table I Study criteria

Inclusion Criteria

I. 18 years of age or older

2. Previous diagnosis of dry eye disease (DED) by an ophthalmologist

3. Persistent signs and symptoms of DED for at least I month

Exclusion Criteria

I. Intraocular surgery or laser within 90 days

2. History of acupuncture treatment within the past year

3. Known history of adverse reaction to acupuncture

4. Pregnancy

5. Breast feeding

to enrollment in the study and were excluded if the test was positive. All participants were counseled to continue their current dry eye regimen as closely as possible. Adverse events and reasons for dropout were recorded.

\section{Study design}

This was a prospective, randomized, double-blinded, shamacupuncture-controlled study. Participants were randomly assigned to a true acupuncture treatment group $(n=24)$ or a sham acupuncture group $(\mathrm{n}=25)$. Randomization was performed with permuted blocks using a randomization table created by http://www.randomization.com. Participants were not informed of which treatment group they belonged to until after the study was concluded. Only the acupuncturist knew to which group each participant belonged. To maintain consistency, only one licensed and experienced acupuncturist performed all of the true and sham acupuncture treatments. Only one blinded investigator performed all of the baseline and follow-up eye examinations.

Prior to acupuncture treatment, a baseline ophthalmic examination was performed in the following order:

1. Ocular Surface Disease Index (OSDI) Questionnaire. The OSDI is a 12-item questionnaire that has been shown to be valid and reliable in assessing the severity of DED. ${ }^{33}$ OSDI responses yield a score from 0 to 100 , with higher numbers indicating more severe dry eye.

2. General Questionnaire assessing hours of computer use, frequency of application of artificial tears, frequency of closing eyes due to intolerable symptoms, and 10 common DED symptoms (discomfort, dryness, scratchiness, burning, stinging, tiredness, redness, watering, blurring, decreased vision), with participantrecorded response choices of "never," "rarely," "sometimes," "frequently," and "constantly." Responses were translated to a numerical scale to facilitate data 
presentation. Assigned numerical scores were $0=$ never, $1=$ rarely, $2=$ =sometimes, 3 =frequently, and $4=$ constantly. The numerical values were then averaged for each treatment group and presented as mean \pm SD, with high scores signifying worse symptoms. Participants were also asked to rate their dryness since initiation of treatments. Response choices were "much better," "better," "same," or "worse." These responses were also assigned a numerical value with $0=$ much better, $1=$ better, $2=$ same, or $3=$ worse. The higher the score, the worse the subjective assessment of dryness.

3. Visual acuity testing with Snellen chart.

4. Tear break-up time (TBUT). A micropipetter (Gilson's P20 PIPETMAN) was used to measure exactly $3.0 \mu \mathrm{l}$ of preservative-free $2 \%$ sodium fluorescein to instill in the eye. Tear film stability was measured by examining the patient at a slit lamp using cobalt blue light. The TBUT was defined as the time, in seconds, after three consecutive complete blinks for dry spots to become visible on the cornea. The time was measured by a stopwatch and rounded to the nearest whole second. A mean of three measurements of each eye was recorded. A TBUT of $<10 \mathrm{~s}$ suggests an unstable tear film, and TBUT is reduced in all forms of DED. ${ }^{34}$

5. Ocular surface grading. Evaluation was performed immediately after TBUT, requiring no additional fluorescein instillation. The fluorescein is rapidly taken up by damaged corneal epithelium, and grading of the staining of the ocular surface has long been used to characterize DED, assess its severity, and monitor the clinical response to therapy. ${ }^{35}$ Using cobalt blue light, a score of 0-3 was given to five sections of the corneal surface (central, inferior, superior, nasal, and lateral) and lateral and nasal parts of the bulbar conjunctiva of each eye, for a maximum total score of 21. A score of 0 was given for no staining; 1 was given for few punctate staining that could be easily counted; 2 was given for moderate punctate staining where the individual punctate staining was too great to count, but had not yet coalesced; 3 was given for coalesced staining.

6. Tear flow assessment using Schirmer test with anesthesia (Topical anesthetic, proparacaine 0.5\%). After instillation of the anesthetic, Schirmer test paper (ColorBar SCHIRMER TEAR TEST; Eagle Vision, Memphis, TN, USA) was placed on the lateral third of the lower eyelid in both eyes for 5 mins, as recorded with a stopwatch. Less than $6 \mathrm{~mm}$ of wetting after 5 mins indicates a diagnosis of teardeficiency. $^{34}$

7. Atmospheric conditions. The mean temperature $\left({ }^{\circ} \mathrm{F}\right)$, dew point $\left({ }^{\circ} \mathrm{F}\right)$, and relative humidity (\%) were recorded for every visit. Atmospheric data was obtained from National Oceanic and Atmospheric Administration, National Weather Service.

True acupuncture was performed per the Niemtzow Protocol. ${ }^{36}$ For the treatment group, each participant received one acupuncture treatment on two consecutive days for a total of two treatment sessions per participant. All acupuncture treatments were performed in a room designed specifically for acupuncture in the UPMC Eye Center's Center for Integrative Eye Care. Acupuncture needs (Seirin, No. 3 gauge $0.20 \mathrm{~mm} \times 30 \mathrm{~mm}$; Seirin-America, Weymouth, MA, USA) were sterile, disposable, and stainless steel, with individual guide tubes that were left in place for 45 mins without electrical or manual stimulation. For true acupuncture treatment, a total of 12 needles were placed bilaterally on the ears (at acupuncture points Salivary Gland 2, Point Zero, and Shen Men) and bilaterally on the index fingers (Large Intestine-1 [LI-1] and Large Intestine 2 [LI-2], and at a point between LI1and LI-2). The acupuncture technique has previously been described for the treatment of xerostomia, ${ }^{24,37,38}$ and published online with modification for use in DED. For sham acupuncture treatment, four needles were placed bilaterally in a $1 \mathrm{~cm}$ circle situated on the left and right upper shoulder areas outside of any known acupuncture points or meridians. Follow-up examinations assessing the same criteria as pre-treatment were performed at one month, three months, and six months after treatment. One week after treatment, participants were also asked to complete both the OSDI questionnaire and General Questionnaire. Figure 1 shows a flow chart of the study design.

\section{Statistical analysis}

A two-way ANOVA analysis and two-sample independent Student's $t$-tests were used for testing intragroup and intergroup hypotheses. Since the experimental design calls for repeated measurements, a two-way ANOVA was the preferred test. Furthermore, using a sham acupuncture control group controlled for the between-subjects effect. Test results were considered to be significant if $p<0.05$. A primary endpoint for this study was the improvement in OSDI. To detect significant improvements in OSDI, 


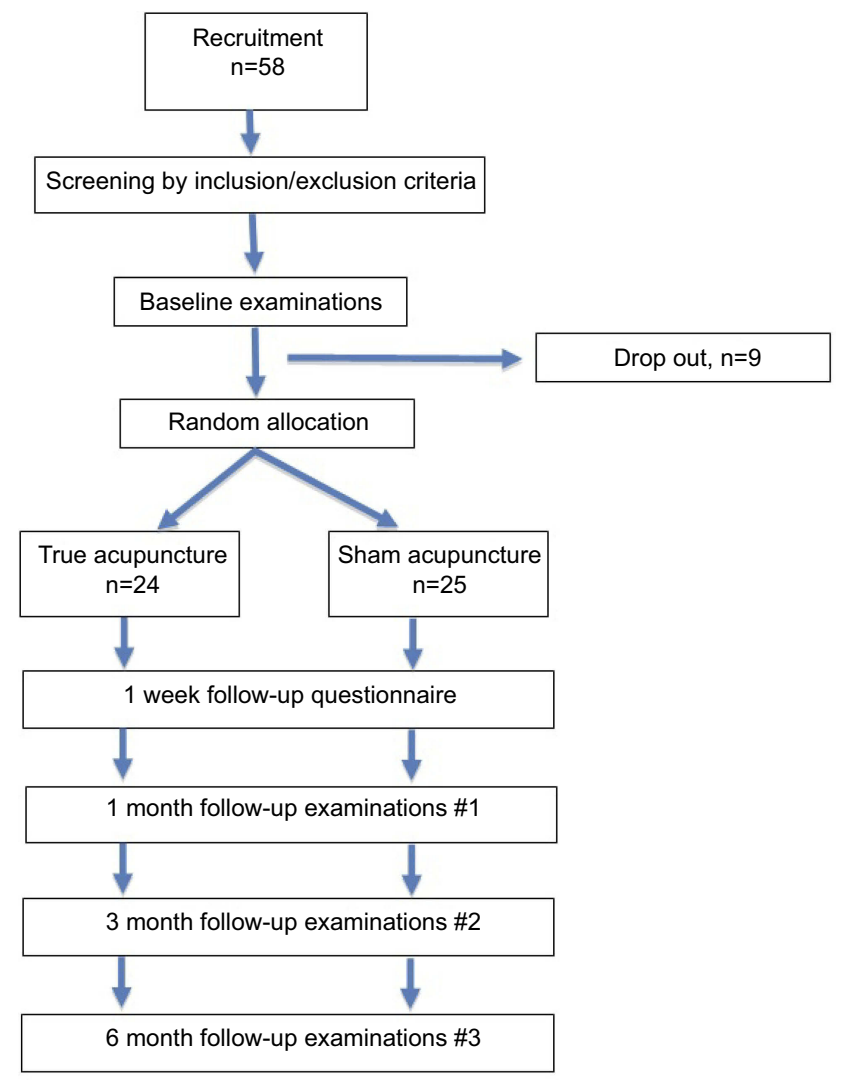

Figure I Schematic diagram of the study design.

historical data was referenced, which suggested a 2.5 unit difference in OSDI means a SD of 2.8 (power $=80 \%$, alpha $=0.05$ ) would require a patient population of at least 20 patients in each group. ${ }^{39}$ For General Questionnaire data, a Kruskal-Wallis test was performed on ranks to achieve statistical differences, as these data do not follow normal distribution. Unless otherwise noted, data are presented as mean $\pm \mathrm{SD}$. All analyses were performed using Prism (GraphPad Software, Inc, La Jolla, CA).

\section{Results}

\section{Participant demographics}

Of the 58 participants recruited in the study, seven dropped out before true or sham acupuncture treatment. Of these, one participant withdrew with no reason stated, while the others withdrew due to pregnancy. A total of 49 patients completed the study.

Participants in the two groups started the study in similar condition. At baseline, the average age of $54 \pm 13$ $(\mathrm{n}=24)(p=0.2)$. There were two more males in the sham acupuncture treatment group than in the true acupuncture group (Table 2). Also, participants' daily computer use,
Table 2 Demographic data and baseline values in true and sham acupuncture treatment groups

\begin{tabular}{|l|l|l|}
\hline Characteristic & $\begin{array}{l}\text { True } \\
\text { acupuncture }\end{array}$ & Sham \\
\cline { 3 - 3 } & Group (n=24) & $\begin{array}{l}\text { Group } \\
(\mathbf{n = 2 5 )}\end{array}$ \\
\hline & & $60 \pm 14$ \\
\hline Age (average) & $54 \pm 13$ & $24-83$ \\
Age range & $24-83$ & 19 \\
Females & 20 & 3 \\
Pre-menopausal & 4 & 16 \\
Post-menopausal & 16 & 6 \\
Males & 4 & $19: 6$ \\
Female/male ratio & $5: 1$ & $4 \pm 4$ \\
Computer use (hours/day) & $4 \pm 3$ & \\
\hline
\end{tabular}

Note: Wherever applicable, data are presented as mean \pm SD.

which could contribute to eyestrain, was $4 \pm 3$ hrs per day in the true acupuncture group and $4 \pm 4$ hrs per day in the sham treatment group $(p=0.6)$.

\section{Atmospheric conditions}

Atmospheric conditions could potentially complicate dry eye assessments and have the potential to confound results. To account for this variable, mean temperature, dew point, and relative humidity were recorded at the time of data collection throughout the study period (Table 3). During the six-month enrollment period, the mean temperature decreased as follow-up time points extended to the fall and winter seasons. In spite of this temperature differential, there were no significant differences between the treatment groups $(p>0.9)$. Similarly, there were no significant differences in dew point and relative humidity between the two treatment groups over the course of the study $(p>0.4)$.

\section{Ocular surface disease index (OSDI)}

OSDI was evaluated at baseline and after 1 week, and 1, 3, and 6 months in patients receiving either a true or sham acupuncture treatment (Figure 2). After true acupuncture treatment, OSDI scores significantly improved from a baseline of $34 \pm 17$ to $19 \pm 17$ ( 1 week, $p<0.01$ ), $21 \pm 17$ (1 month, $p<0.05$ ), $20 \pm 21$ ( 3 months, $p<0.05$ ), and $16 \pm 12$ (6 months, $\mathrm{n}=24, p<0.01)$. Although the OSDI scores improved from $36 \pm 20$ after sham treatment to $24 \pm 22$ (1 week), $24 \pm 21$ (1 month), $21 \pm 20$ (3 months), and 25 \pm 18 (6 months), none were significant when compared to baseline $(\mathrm{n}=25, p=0.09)$. OSDI scores improved in both groups after 1 week, and 
Table 3 Atmospheric conditions at evaluation points throughout the study

\begin{tabular}{|c|c|c|c|c|c|}
\hline & Treatment & Baseline & I Month & 3 Month & 6 Month \\
\hline \multirow[t]{2}{*}{ Mean temperature $\left({ }^{\circ} \mathrm{F}\right)$} & $\mathrm{T}$ & $60 \pm 15$ & $59 \pm 16$ & $49 \pm 17$ & $39 \pm 17$ \\
\hline & $\mathrm{S}$ & $60 \pm 12$ & $61 \pm 16$ & $46 \pm 17$ & $38 \pm 15$ \\
\hline \multirow{3}{*}{$\begin{array}{l}\text { Dew } \\
\text { point }\left({ }^{\circ} \mathrm{F}\right)\end{array}$} & $\mathrm{T}$ & $47 \pm 15$ & $48 \pm 16$ & $39 \pm 19$ & $27 \pm 16$ \\
\hline & & & & & \\
\hline & $S$ & $49 \pm 12$ & $48 \pm 17$ & $33 \pm 16$ & $28 \pm 16$ \\
\hline \multirow[t]{2}{*}{ Relative humidity (\%) } & $\mathrm{T}$ & $64 \pm 13$ & $68 \pm 18$ & $7 I \pm 22$ & $65 \pm 20$ \\
\hline & $\mathrm{S}$ & $67 \pm 13$ & $64 \pm 13$ & $63 \pm 315$ & $70 \pm 21$ \\
\hline
\end{tabular}

Note: Data are presented as mean \pm SD.

Abbreviations: T, true treatment; $\mathrm{S}$, sham treatment.

\section{Ocular Surface Disease Index (OSDI)}

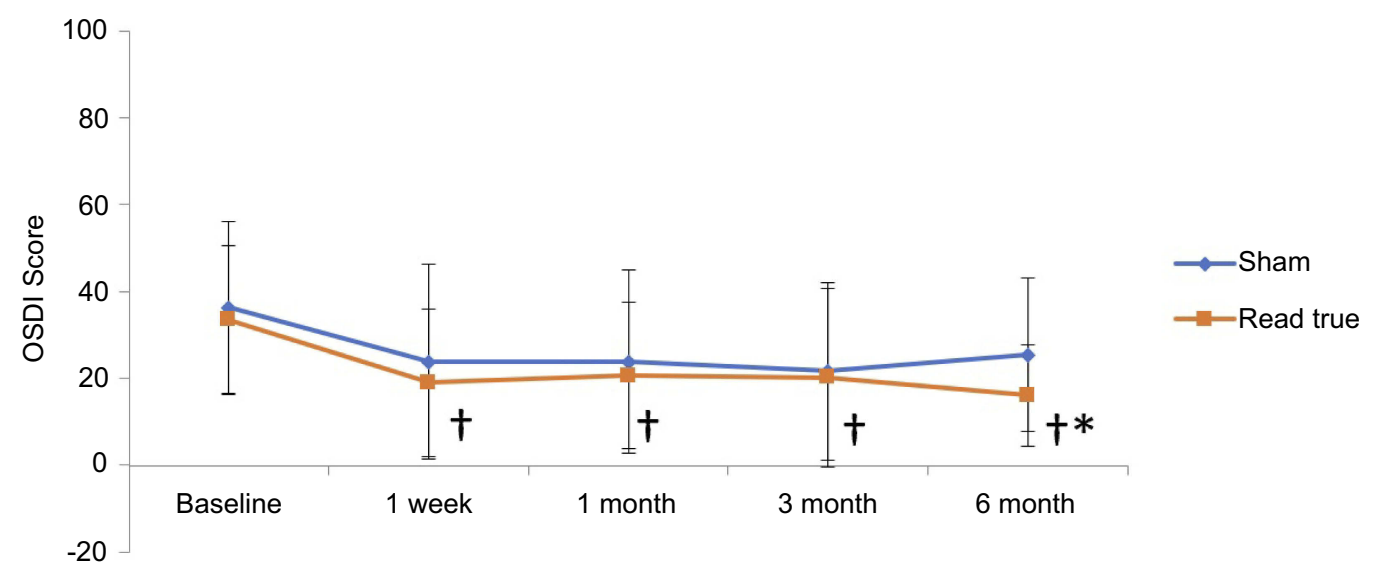

Figure 2 Ocular Surface Disease Index (OSDI). OSDI was evaluated at baseline and after I week, I, 3, and 6 months in patients receiving either true $(\mathrm{n}=24)$ or sham ( $\mathrm{n}=25$ ) acupuncture treatment. True acupuncture treatment significantly improved OSDI when compared to sham treatment only after 6 months $\left({ }^{*} p=0.04\right)$. Also, OSDI scores significantly improved I week after true acupuncture treatment and continued to improve $(\dagger p<0.05)$.

continued to improve in the treatment group throughout the duration of the study $(p<0.05)$. At 6 months after treatment, true acupuncture treatment significantly improved OSDI scores compared to sham treatment $(p=0.04)$.

\section{Secondary outcome measures}

Secondary outcome assessment measures were recorded during the study period and results presented as mean \pm $\mathrm{SD}$ (Table 4). After 6 months, a significant reduction in the

Table 4 Secondary outcomes were evaluated at I, 3 and 6 months

\begin{tabular}{|l|l|l|l|l|l|l|l|}
\hline Outcome measure & Treatment & Baseline & I Month & $\mathbf{3}$ Month & $\mathbf{6}$ Month & $\mathbf{N}$ & $\mathbf{p}$-values $* / * * / * * *$ \\
\hline TBUT & $\mathrm{T}$ & $3 \pm 3$ & $3 \pm 3$ & $3 \pm 3$ & $3 \pm 3$ & 24 & $<0.9 / 0.5 / 0.3$ \\
Schirmer's test & $\mathrm{S}$ & $3 \pm 2$ & $3 \pm 3$ & $3 \pm 3$ & $2 \pm 2$ & 25 & \\
& $\mathrm{~T}$ & $10 \pm 8$ & $9 \pm 6$ & $10 \pm 7$ & $11 \pm 9$ & 24 & $<0.3 / 0.4 / 0.3$ \\
Ocular surface grading & $\mathrm{S}$ & $9 \pm 7$ & $1 \mathrm{I} \pm 7$ & $8 \pm 4$ & $10 \pm 6$ & 25 & \\
& $\mathrm{~T}$ & $8 \pm 4$ & $6 \pm 4$ & $7 \pm 4$ & $7 \pm 4$ & 24 & $<0.5 / 0.1 / 0.4$ \\
Artificial tear application (questionnaire) & $\mathrm{S}$ & $6 \pm 3$ & $5 \pm 3$ & $6 \pm 3$ & $6 \pm 2$ & 25 & \\
& $\mathrm{~T}$ & $3 \pm 3$ & $3 \pm 3$ & $3 \pm 3$ & $2 \pm 2$ & 24 & $<0.5 / 0.3 / 0.3$ \\
Frequency of eye closing (questionnaire) & $\mathrm{T}$ & $4 \pm 3$ & $3 \pm 3$ & $3 \pm 2$ & $3 \pm 3$ & 25 & \\
& $\mathrm{~S}$ & $6 \pm 4$ & $3 \pm 4$ & $3 \pm 5$ & $3 \pm 4$ & 24 & $<0.2 / 0.002 / 0.2$ \\
& $5 \pm 4$ & $3 \pm 3$ & $4 \pm 4$ & $3 \pm 3$ & 25 & \\
\hline
\end{tabular}

Notes: *Comparison of True vs Sham treatment groups. **Compared to baseline within the True treatment group. ***Compared to baseline within the Sham treatment group. Data are presented as mean \pm SD.

Abbreviations: T, true treatment; S, sham treatment; TBUT, tear film break-up time. 
patient-reported frequency of eye closing was observed among participants receiving true acupuncture treatment when compared to baseline ( $p=0.002, \mathrm{n}=24)$, as recorded in the General Questionnaire. Although there was a reduction in the frequency of eye closing in the sham-treated participants, the difference was not significant $(p=0.2, \mathrm{n}=25)$. Furthermore, there was a trend towards decreased number of artificial tear applications in the true and sham acupuncture groups, however, the change did not reach statistical significance. For both true and sham acupuncture treatment groups, there were no significant differences in TBUT, Schirmer's test, and ocular surface grading at 1 month, 3 months, and 6 months after treatment (Table 4).

\section{General questionnaire symptoms}

At baseline and at each follow-up visit, the participants in each group completed a general questionnaire assessing symptoms over the past week (Table 5). Responses were assigned numerical scores, $0=$ never, $1=$ rarely, $2=$ sometimes, $3=$ frequently, and $4=$ constantly. The numerical values were then averaged for each treatment group and presented as mean $\pm \mathrm{SD}$, with higher scores representing worse symptoms. Baseline characteristics for all DED symptoms in both true or sham treatment groups were similar $(p>0.2)$. Participants reported a significant reduction in discomfort, dryness, and scratchiness at 1 month after true acupuncture treatment and remained decreased throughout the study period. Patients in the sham acupuncture group also reported a significant reduction in discomfort and scratchiness, but this effect was reported 3 months after treatment. At 6 months, there was a statistically significant decrease in intragroup analysis of true acupuncture group in dryness $(p=0.001)$, scratchiness $(p=0.001)$, and redness $(p=0.01)$. While a significant improvement in dryness was reported at 1 month after either true or sham treatments, the effect was not sustained in the sham treatment group at 6 months. There were no significant improvements in other DED symptoms such as burning, stinging, watering, blurring, and decreased vision after either true or sham acupuncture throughout the study period. When asked to rate the improvement in dryness at 6 months after initiation of treatment, the participants reported a score of $1 \pm 1$ after true acupuncture treatment and $2 \pm 1$ after sham acupuncture treatment. These numbers translate to same or better rating with no significant differences between the two treatments $(p=0.2)$.

Table 5 General questionnaire DED symptoms score

\begin{tabular}{|c|c|c|c|c|c|c|c|}
\hline DED symptoms & Treatment & Baseline & I Month & 3 Month & 6 Month & $\mathbf{N}$ & p-Values $* / * * / * * *$ \\
\hline \multirow[t]{2}{*}{ Discomfort } & $\mathrm{T}$ & $2 \pm 1$ & $1 \pm 1 \dagger$ & $1 \pm 1 \dagger$ & $1 \pm 1 \dagger$ & 24 & $>0.9 / 0.01 / 0.02$ \\
\hline & $S$ & $2 \pm 1$ & $2 \pm 1$ & $I \pm I \dagger$ & $I \pm 1 \dagger$ & 25 & \\
\hline \multirow[t]{2}{*}{ Dryness } & $\mathrm{T}$ & $3 \pm 1$ & $2 \pm 1 \dagger$ & $2 \pm 1 \dagger$ & $I \pm I t$ & 24 & $>0.9 / 0.001 / 0.01$ \\
\hline & $S$ & $3 \pm 1$ & $2 \pm 1 \dagger$ & $2 \pm 1 \dagger$ & $2 \pm 1 \dagger$ & 25 & \\
\hline \multirow[t]{2}{*}{ Scratchiness } & $\mathrm{T}$ & $2 \pm 1$ & $I \pm I t$ & $I \pm I \dagger$ & $I \pm I t$ & 24 & $>0.9 / 0.001 / 0.3$ \\
\hline & $S$ & $2 \pm 1$ & $\mathrm{I} \pm \mathrm{I}$ & $I \pm I \dagger$ & $I \pm I t$ & 25 & \\
\hline \multirow[t]{2}{*}{ Burning } & $\mathrm{T}$ & $I \pm I$ & $I \pm I$ & $I \pm I$ & $I \pm I$ & 24 & $>0.6 / 0.1 / 0.1$ \\
\hline & $S$ & $2 \pm 2$ & $\mathrm{I} \pm 2$ & $I \pm I$ & $I \pm I$ & 25 & \\
\hline \multirow[t]{2}{*}{ Stinging } & $\mathrm{T}$ & $I \pm I$ & $I \pm I$ & $I \pm I$ & $I \pm I$ & 24 & $>0.9 / 0.1 / 0.3$ \\
\hline & $S$ & $I \pm I$ & $I \pm I$ & $I \pm I$ & $I \pm I$ & 25 & \\
\hline \multirow[t]{2}{*}{ Tiredness } & $\mathrm{T}$ & $2 \pm 1$ & $I \pm I$ & $\mathrm{I} \pm 2$ & $1 \pm 1 \dagger$ & 24 & $>0.4 / 0.1 / 0.2$ \\
\hline & $S$ & $2 \pm 1$ & $2 \pm 2$ & $2 \pm 1$ & $2 \pm 1$ & 25 & \\
\hline \multirow[t]{2}{*}{ Redness } & $\mathrm{T}$ & $2 \pm 1$ & $I \pm I t$ & $I \pm I$ & $I \pm 1 \dagger$ & 24 & $>0.3 / 0.01 / 0.1$ \\
\hline & $\mathrm{S}$ & $2 \pm 2$ & $\mathrm{I} \pm 2$ & $I \pm I$ & $I \pm 2$ & 25 & \\
\hline \multirow[t]{2}{*}{ Watering } & $\mathrm{T}$ & $I \pm I$ & $I \pm I$ & $I \pm I$ & $0 \pm 1$ & 24 & $>0.2 / 0.4 / 0.5$ \\
\hline & $S$ & $I \pm I$ & $I \pm I$ & $I \pm I$ & $I \pm I$ & 25 & \\
\hline \multirow[t]{2}{*}{ Blurring } & $\mathrm{T}$ & $I \pm I$ & $I \pm I$ & $I \pm I$ & $I \pm I$ & 24 & $>0.4 / 0.1 / 0.1$ \\
\hline & $S$ & $2 \pm 2$ & $I \pm I$ & $I \pm I$ & $I \pm I$ & 25 & \\
\hline \multirow[t]{2}{*}{ Decreased Vision } & $\mathrm{T}$ & $I \pm I$ & $I \pm I$ & $I \pm I$ & $I \pm I$ & 24 & $>0.5 / 0.6 / 0.2$ \\
\hline & $S$ & $\mathrm{I} \pm 2$ & $I \pm I$ & $I \pm I$ & $I \pm I$ & 25 & \\
\hline
\end{tabular}

Notes: *Comparison of True vs Sham treatment groups. **Compared to baseline within the True treatment group. ***Compared to baseline within the Sham treatment group. $\dagger$ Intragroup comparison to baseline. Responses were assigned numerical scores, $0=$ never, I=rarely, 2=sometimes, $3=$ frequently and $4=$ constantly. The higher the score the worse the symptoms. A Kruskal-Wallis test was performed on ranks to achieve statistical differences, as these data do not follow normal distribution. Abbreviations: T, true treatment; $S$, sham treatment; DED, dry eye disease. 


\section{Adverse effects}

No adverse effects of true or sham acupuncture were noted.

\section{Discussion}

Our prospective, randomized, double-blinded shamacupuncture-controlled study suggests that two treatment sessions on consecutive days of true acupuncture can improve subjective outcomes measures of dry eye symptoms, as assessed by OSDI and a general questionnaire. Also, of note, OSDI scores were reduced in both groups at 1 week after acupuncture treatment, but at 6 months after treatment there was a statistically significant improvement in the treatment group compared to the sham acupuncture group. Furthermore, the subjective improvements of dryness, scratchiness, and redness were significant in intragroup analysis of the true treatment group, but not the sham acupuncture group.

Several mechanisms of action of acupuncture have been proposed. Acupuncture has been found to decrease proinflammatory cytokines, thereby having an anti-inflammatory effect. This has implications for chronic inflammatory conditions such as inflammatory bowel disease, dry eye, rheumatoid arthritis, and depression. ${ }^{40-42}$ Furthermore, acupuncture has been found to increase blood flow to organs, ${ }^{43}$ reduce the sensation of pain, ${ }^{44,45}$ and modulate the sympathetic system in mechanisms similar to muscle contraction in prolonged exercise. ${ }^{46} \mathrm{~A}$ series of studies analyzed protein expression in blood, urine, and saliva before and after stimulation of the Zusanli (ST-36) point, a point commonly used to treat a wide range of gastrointestinal disorders. The authors concluded that stimulation of the acupoint led to activation of different metabolic pathways. ${ }^{47-49}$ The effects of acupuncture have been further validated through neuroimaging studies. Dhond et al. reported functional magnetic resonance imaging studies in humans which showed immediate effects of prolonged acupuncture stimulation in the limbic and basal forebrain areas related to somatosensory and affective functions known to be involved in pain processing. ${ }^{50}$ Harris et al. documented that positron-emission tomography has shown acupuncture to increase $\mu$-opioid-binding potential for several days in some of the same brain areas, while also showing distinct changes in limbic structures between true and sham acupuncture. $^{51}$

Our study suggests that the mechanism of action of acupuncture for dry eye may be due more to modulation of pain intensity or threshold rather than modulation of lacrimal gland function. We found no improvement in tear flow, TBUT, or ocular surface staining, which are more objective indicators of DED. However, consistent with a mechanism that modulates pain intensity or threshold, we found statistically significant improvements in DED symptoms in both treatment groups at 1 week after treatment.

Interestingly, several randomized control trials comparing acupuncture to artificial tears showed statistically significant improvements on parameters of dry eye, but the effect can be delayed..$^{20,22,23,25,29,48}$ Gong et al. showed that there were significant differences in the acupuncture group compared to the artificial tears control group in symptoms, TBUT, and Schirmer test scores at 3 weeks post-treatment, but not immediately post-treatment. ${ }^{20}$ Furthermore, Kim et al. showed no improvements in the acupuncture compared to the artificial tear group immediately after treatment, but significant improvement in OSDI and visual analog scale (VAS, self-assessment for ocular discomfort) in the acupuncture group 8 weeks after the end of acupuncture treatment. ${ }^{22}$ Similarly, our study found OSDI scores to be similar between true acupuncture and sham groups until 6 months after treatment, suggesting a delayed treatment effect of the true acupuncture group.

A meta-analysis by Yang et al. performed on data from seven randomized control trials comparing acupuncture with artificial tear replacement found that acupuncture therapy improved TBUT, Schirmer I test, corneal fluorescein staining, and VAS compared with artificial tears. ${ }^{28}$ Another systematic review by Lee et al. concluded that all prior acupuncture studies had high risk of bias, due to lack of blinding, insufficient sample size, and no power analysis. ${ }^{52}$ The authors also point out that not a single acupuncture study has reported negative results and therefore limited ability to draw reliable conclusions.

The strength in our study lies in the fact that we conducted a prospective double-blinded, randomized controlled trial, using sham acupuncture as the control. This thereby quantifies potential biases and placebo effect. Furthermore, we are one of the only studies to include a power analysis. To our knowledge, only one other study by Shin et al. have evaluated the efficacy of acupuncture for DED using sham acupuncture as a control. ${ }^{32}$ After subjects completed three treatment sessions per week over a 3-week study period, the authors found no statistically significant differences in OSDI, TBUT, or Schirmer's test from baseline between true and sham acupuncture groups. However, within-group analysis showed improvements in OSDI and VAS in both treatment 
and sham groups and TBUT in the treatment group at 3 weeks after treatment. Our results of finding a statistically significant decrease in OSDI between true and sham acupuncture groups can possibly be explained by the difference in acupuncture methodology. Compared to Shin et al, we used different meridians for both true and sham acupuncture, and we did not use "de qi" manipulation. Furthermore, we included a longer follow-up period of 6 months compared to 4-week analysis that was used by Shin and colleagues. Because the mechanism of action of acupuncture is still widely unknown, it is possible that our method is more efficacious for the treatment of DED symptoms than the currently published studies or that the longer follow-up period captured the delayed effects of acupuncture. It is also possible that sham acupuncture can lead to unintended treatment effects. In fact, Lund et al. argue that sham acupuncture is not acceptable as a placebo control because even superficial minimal sham acupuncture procedure can lead to physiologic change beyond that of a placebo effect. ${ }^{53,54}$

There are a few limitations in our study which the authors would like to highlight. First, our study design included all types of dry eye, including aqueous deficiency (ie, Sjogren's) and evaporative dry eye (ie, rosacea). Given the Niemtzow Protocol was designed to treat both xerostomia and DED, it is possible that Sjogren's dry eye patients may have further benefit from the treatment. Furthermore, the study did not control for use of medications for dry eye, contact lens use, medical comorbidities, or patient demographic information such as ethnicity. All of these coule be potential confounding factors that could influence documented treatment effect.

In a recent systematic review, Kim et al. concluded that longer periods of treatment ( $>1$ month) was preferable to shorter periods, and that there was an optimum number of two to three space-out treatments per week. ${ }^{55}$ While our study only included two treatments on consecutive days, it is possible there could be a greater treatment effect had these patients received treatments over many weeks. Furthermore, there is no accepted acupuncture for dry eye regimen and most published studies apply needles to different sets of acupoints. More research is needed to create an optimized treatment protocol for acupuncture for dry eye.

\section{Conclusion}

Our study shows improvement in dry eye symptoms using true acupuncture compared to sham acupuncture for at least 6 months post-treatment. Specifically, there was a significant decrease between true and sham treatment groups in OSDI scores at 6 months, and intragroup significant decrease in symptoms of dryness, scratchiness, and redness in the true acupuncture group. Although this did not reach statistical significance, patients who received true acupuncture treatment required fewer artificial tear drops than those who received sham acupuncture, which aids to decrease the financial burden of dry eye. Acupuncture can be a useful adjunctive treatment to effectively reduce dry eye symptoms, especially given the lowrisk profile. While further investigation is needed to optimize an acupuncture treatment regimen, our study suggests that acupuncture has an effect on dry eye symptoms beyond a placebo effect.

\section{Acknowledgments}

The authors would like to acknowledge Theresa DiCaprio for her help with recruitment, scheduling, and general assistance with this project. We further would like to recognize funding from National Institutes of Health CORE Grant P30 EY008098; Eye and Ear Foundation of Pittsburgh, PA; and an Unrestricted Grant from Research to Prevent Blindness, New York, NY.

\section{Disclosure}

Deepinder K Dhaliwal, MD, LAc is currently receiving support from the National Institutes of Health core grant (P30 EY008098), Eye and Ear Foundation of Pittsburgh, PA, and an unrestricted grant from Research to Prevent Blindness, New York, NY. The other authors report no conflicts of interest in this work.

\section{References}

1. Saaddine JB, Venkat Narayan K, Vinicor F. Vision loss: a public health problem? Ophthalmology. 2003;110(2):253-254. doi:10.1016/S01616420(02)01839-0

2. Schein OD, Munuz B, Tielsch JM, Bandeen-Roche K, West S. Prevalence of dry eye among the elderly. Am $J$ Ophthalmol. 1997;124(6):723-728.

3. The definition and classification of dry eye disease: report of the definition and classification subcommittee of the international dry eye workshop (2007). Ocul Surf. 2007;5(2):75-92. doi:10.1016/ S1542-0124(12)70081-2

4. Schaumberg DA, Sullivan DA, Buring JE, Dana MR. Prevalence of dry eye syndrome among US women. Am J Ophthalmol. 2003;136 (2):318-326. doi:10.1016/S0002-9394(03)00218-6

5. Perry HD. Dry eye disease: pathophysiology, classification, and diagnosis. Am J Manag Care. 2008;14(3 Suppl):79-87.

6. Tavares FP. Dry eye disease. Semin Ophthalmol. 2010;25(3):84-93. doi:10.3109/08820538.2010.488568 
7. Calonge M. The treatment of dry eye. Surv Ophthalmol. 2001;45 (SUPPL. 2):S227-S239. doi:10.1016/S0039-6257(00)00205-8

8. Lemp MA. Management of dry eye disease. Am J Manag Care. 2008;14(SUPPL.3):S88-S101. Available from: http://ovidsp.ovid com/ovidweb.cgi? $\mathrm{T}=\mathrm{JS} \& \mathrm{PAGE}=$ reference $\& \mathrm{D}=$ emed $8 \& \mathrm{NEWS}=$ $\mathrm{N} \& A N=2008228314$.

9. Lemp MA. Advances in understanding and managing dry eye disease. Am J Ophthalmol. 2008;146(3):350-356.e1. doi:10.1016/j. ajo.2008.05.016

10. Mandal P, Khan MA, Shah S. Contact lens and anterior eye drugs do we need them? Applications of non-pharmaceutical therapy in anterior eye disease : a review. Cont Lens Anterior Eye. 2018;40 (6):360-366. doi:10.1016/j.clae.2017.09.001

11. Friedman NJ. Impact of dry eye disease and treatment on quality of life. Curr Opin Ophthalmol. 2010;21(4):310-316. doi:10.1097/ ICU.0b013e32833a8c15

12. Brown MM, Brown GC, Brown HC, Peet J, Roth Z. Value-based medicine, comparative effectiveness, and cost-effectiveness analysis of topical cyclosporine for the treatment of dry eye syndrome. Arch Ophthalmol. 2009;127(2). doi:10.1001/archop hthalmol.2008.608

13. Sierpina VS, Frenkel MA. Acupuncture: a clinical review. South Med J. 2005;98(3):330-337. doi:10.1097/01.SMJ.0000140834.30654.0F

14. White A, Ernst E. A brief history of acupuncture. Rheumatology. 2004;43(5):662-663. doi:10.1093/rheumatology/keg005

15. Ernst E. Acupuncture - a critical analysis. J Intern Med. 2006;259 (2):125-137. doi:10.1111/j.1365-2796.2005.01584.x

16. Rhee DJ, Spaeth GL, Myers JS, et al. Prevalence of the use of complementary and alternative medicine for glaucoma. Ophthalmology. 2002;109:438-443.

17. Smith JR, Spurrier NJ, Martin JT, Rosenbaum JT. Prevalent use of complementary and alternative medicine by patients with inflammatory eye disease. Ocul Immunol Inflamm. 2004;12(3):203-214. doi:10.1080/092739490500200

18. Eliason KJ, Richards SC, Schamaun GT. Acupuncture treatment for dry eye. Med Acupunct. 2007;19(1):25-28. doi:10.1089/acu. 2006.504

19. Gong L, Sun X. Treatment of intractable dry eyes: tear secretion increase and morphological changes of the lacrimal gland of rabbit after acupuncture. Acupunct Electro-Therapeutics Res. 2007;32(3-4):223-233. doi:10.3727/036012907815844011

20. Gong L, Sun X, Chapin WJ. Clinical curative effect of acupuncture therapy on xerophthalmia. Am J Chin Med. 2010;38(4):651-659. doi:10.1142/S0192415X10008123

21. Jeon J-H, Shin M-S, Lee MS, et al. Acupuncture reduces symptoms of dry eye syndrome: a preliminary observational study. J Altern Complement Med. 2010;16(12):1291-1294. doi:10.1089/acm. 2009.0537

22. Kim TH, Kang JW, Kim KH, et al. Acupuncture for the treatment of dry eye: a multicenter randomised controlled trial with active comparison intervention (artificial teardrops). PLoS One. 2012;7 (5): $1-9$.

23. Lin T, Gong L, Liu X, Ma X. Fourier-domain optical coherence tomography for monitoring the lower tear meniscus in dry eye after acupuncture treatment. Evidence-Based Complement Altern Med. 2015;2015:1-10. doi:10.1155/2015/492150

24. Johnstone PAS, Niemtzow RC, Riffenburgh RH. Acupuncture for xerostomia: clinical update. Cancer. 2002;94(4):1151-1156.

25. Nepp J, Wedrich A, Akramian J, et al. Dry eye treatment with acupuncture. A prospective, randomized, double-masked study. $A d v$ Exp Med Biol. 1998;438:1011-1016.

26. Qiu X, Gong L, Sun X, Guo J, Chodara AM. Efficacy of acupuncture and identification of tear protein expression changes using iTRAQ quantitative proteomics in rabbits. Curr Eye Res. 2011;36 (10):886-894. doi:10.3109/02713683.2011.601843
27. Tseng K-L, Liu H-J, Tso K-Y, Woung L-C, Su Y-C, Lin J-G A clinical study of acupuncture and SSP (Silver Spike Point) electro-therapy for dry eye syndrome. Am J Chin Med. 2006;34 (2):197-206. doi:10.1142/S0192415X0600376X

28. Yang L, Yang Z, Yu H, Song H. Acupuncture therapy is more effective than artificial tears for dry eye syndrome: evidence based on a meta-analysis. Evidence-Based Complement Altern Med. 2015;2015:1-11. doi:10.1155/2015/143858

29. Grönlund MA, Stenevi U, Lundeberg T. Acupuncture treatment in patients with keratoconjunctivitis sicca: a pilot study. Acta Ophthalmol Scand. 2004;82(3 Pt 1):283-290. doi:10.1111/j.16000420.2004.00254.x

30. List T, Lundeberg T, Lundström I, Lindström F, Ravald N. The effect of acupuncture in the treatment of patients with primary Sjögren's syndrome. A controlled study. Acta Odontol Scand. 1998;56(2):95-99.

31. Jiang Q, Zhang H, Pang R, Chen J, Liu Z, Zhou X. Acupuncture for Primary Sjögren Syndrome (pSS) on symptomatic improvements : study protocol for a randomized controlled trial. BMC Complement Altern Med. 2017;17:1-8.

32. Shin MS, Kim JI, Lee MS, et al. Acupuncture for treating dry eye: a randomized placebo-controlled trial. Acta Ophthalmol. 2010;88 (8):328-333. doi:10.1111/j.1755-3768.2010.02027.x

33. Schiffman RM, Christianson MD, Jacobsen G, Hirsch JD, Reis BL. Reliability and validity of the ocular surface disease index. Arch Ophthalmol. 2000;118(5):615-621.

34. Bron AJ. Diagnosis of dry eye. Surv Ophthalmol. 2001;45(Suppl 2): S221-S226.

35. Bron AJ, Evans VE, Smith JA. Grading of corneal and conjunctival staining in the context of other dry eye tests. Cornea. 2003;22 (7):640-650

36. Niemtzow RC. Dry mouth and dry eyes acupuncture technique upgrade; 2009. Available from: http://www.n5ev.com/uploads/3/0/9/ 7/30974833/dry_mouth_and_dry_eyes_acupuncture_technique_ update_oct_15_2009.pdf. Accessed January 27, 2019

37. Niemtzow RC, Mb C, Py P, Jp A. Acupuncture technique for pilocarpine-resistant xerostomia following radiotherapy for head and neck malignancies. Med Acupunct. 2000;12:42-43.

38. Johnstone PAS, Peng YP, May BC, Inouye WS, Niemtzow RC. Acupuncture for pilocarpine-resistant xerostomia following radiotherapy for head and neck malignancies. Int $J$ Radiat Oncol Biol Phys. 2001;50(2):353-357.

39. Shiffman R, Christianson M, Jacobsen G, Hirsch J, Reis B. Reliability and validity of the ocular surface disease index. JAMA Ophthalmol. 2000;118(5):615-621.

40. Lu J, Shao R-H, Hu L, Tu Y, Guo J-Y. Potential antiinflammatory effects of acupuncture in a chronic stress model of depression in rats. Neurosci Lett. 2016;618:31-38. doi:10.1016/j.neulet.2016.02.040

41. Oke SL, Tracey KJ. The inflammatory reflex and the role of complenetary and alternative medical therapies. Ann N Y Acad Sci. 2009; (1172):172-180. doi:10.1196/annals.1393.013

42. Kavoussi B, Ross BE. The neuroimmune basis of anti-inflammatory acupuncture. Integr Cancer Ther. 2007;6(3):251-257. doi:10.1177/ 1534735407305892

43. Uchida S, Hotta H. Acupuncture affects regional blood flow in various organs. Evidence-Based Complement Altern Med. 2008;5 (2):145-151. doi:10.1093/ecam/nem051

44. Bäcker M, Grossman P, Schneider J, et al. Acupuncture in migraine: investigation of autonomic effects. Clin J Pain. 2008;24(2):106-115. doi:10.1097/AJP.0b013e318159f95e

45. Nepp J, Jandrasits K, Schauersberger J, et al. Is acupuncture a useful tool for pain-treatment in ophthalmology? Acupunct Electrother Res. 2002;27(3-4):171-182.

46. Andersson S, Lundeberg T. Acupuncture - from empiricism to science: functional background to acupuncture effects in pain and disease pain and disease. Med Hypotheses. 1995;45(3):271-281. 
47. Yan G, Zhang A, Sun H, et al. Dissection of biological property of chinese acupuncture point zusanli based on long-term treatment via modulating multiple metabolic pathways. Evidence-Based Complement Altern Med. 2013;2013:1-10. doi:10.1155/2013/429703

48. Zhang Y, Yang W. Effects of acupuncture and moxibustion on tear-film of the patients with xerophthalmia. J Tradit Chin Med. 2007;27(4):258-260.

49. Zhang Y, Zhang A, Yan G, et al. High-throughput metabolomic approach revealed the acupuncture exerting intervention effects by perturbed signatures and pathways. Mol Biosyst. 2014;10(1):65-73. doi:10.1039/c3mb70352e

50. Dhond RP, Kettner N, Napadow V. Neuroimaging acupuncture effects in the human brain. J Altern Complement Med. 2007;13 (6):603-616. doi:10.1089/acm.2007.7040

51. Harris RE, Zubieta JK, Scott DJ, Napadow V, Gracely RH, Clauw DJ. Traditional Chinese acupuncture and placebo (sham) acupuncture are differentiated by their effects on mu-opioid receptors (MORs). Neuroimage. 2009;47(3):1077-1085. doi:10.1016/j. neuroimage.2009.05.083
52. Lee MS, Shin BC, Choi TY, Ernst E. Acupuncture for treating dry eye: a systematic review. Acta Ophthalmol. 2011;89(2):101-106. doi:10.1111/j.1755-3768.2009.01855.x

53. Lund I, Näslund J, Lundeberg T. Minimal acupuncture is not a valid placebo control in randomised controlled trials of acupuncture: a physiologist's perspective. Dtsch Zeitschrift fur Akupunkt. 2009;52(2):55-56. doi:10.1016/j.dza.2009.04.003

54. Lund I, Lundeberg T. Are minimal, superficial or sham acupuncture procedures acceptable as inert placebo controls? Acupunct Med. 2006;24(1):13-15.

55. Kim BH, Kim MH, Kang SH, Nam HJ. Optimizing acupuncture treatment for dry eye syndrome : a systematic review. $B M C$ Complement Altern Med. 2018;18:1-9.
Clinical Ophthalmology

\section{Publish your work in this journal}

Clinical Ophthalmology is an international, peer-reviewed journal covering all subspecialties within ophthalmology. Key topics include: Optometry; Visual science; Pharmacology and drug therapy in eye diseases; Basic Sciences; Primary and Secondary eye care; Patient Safety and Quality of Care Improvements. This journal is indexed on PubMed

\section{Dovepress}

Central and CAS, and is the official journal of The Society of Clinical Ophthalmology (SCO). The manuscript management system is completely online and includes a very quick and fair peer-review system, which is all easy to use. Visit http://www.dovepress.com/ testimonials.php to read real quotes from published authors. 